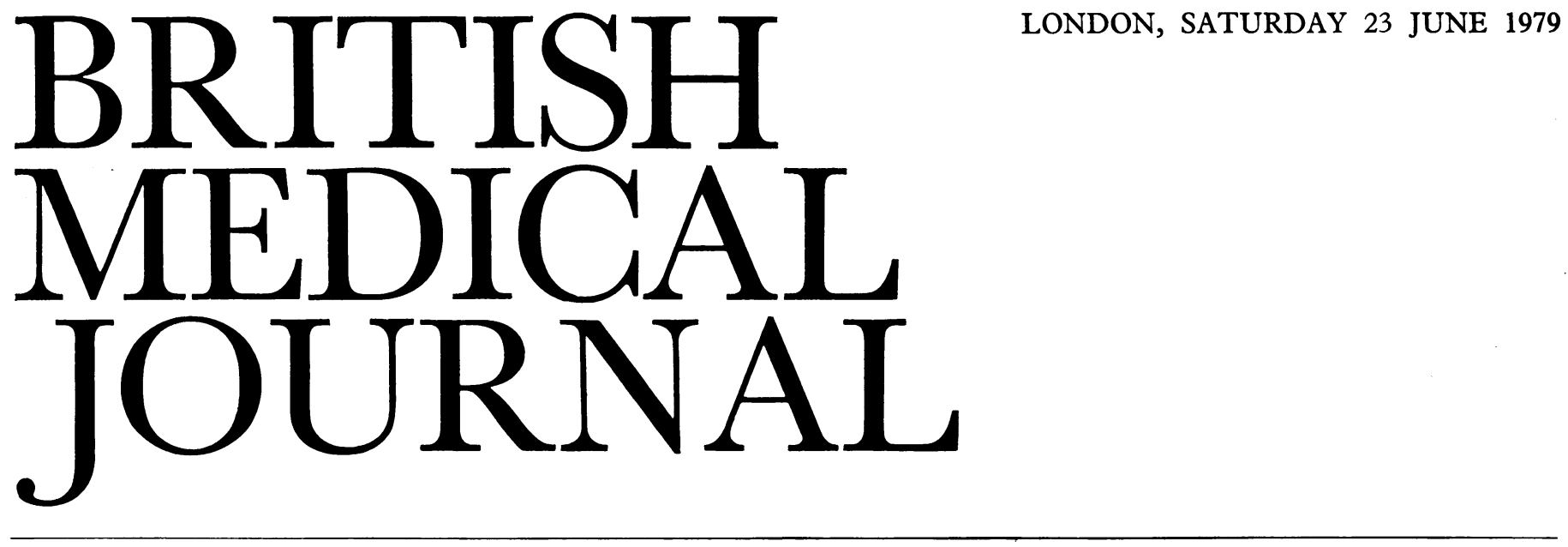

\title{
Thromboembolism in pregnancy
}

The most dreaded complication of venous thrombosis in pregnancy is pulmonary embolism, which is second only to abortion as a cause of maternal mortality. ${ }^{1}$ If that risk is avoided chronic venous insufficiency due to untreated thrombosis may result in an unsightly and painful leg in which future thrombi are likely. But, while accurate diagnosis and prompt treatment are essential in pregnancy, the diagnostic methods and treatments in current use have dangers and limitations.

When a pregnant woman presents with signs of venous thrombosis treatment should be started at once with heparin by intravenous infusion while the diagnosis is being confirmed. The problem is that clinical diagnosis is unreliable, ${ }^{2}$ and radioactive fibrinogen uptake methods are unacceptable in pregnancy. ${ }^{3}$ Doppler ultrasound has its limitations but is useful and readily available for detecting major vein thrombosis, ${ }^{4}$ and thermographic techniques and impedance phlebography await full assessment in pregnancy. Radionuclide venography is similar in its accuracy to contrast phlebography," and if-as seems likely-the radiation risk to the fetus is small, it may prove to be the most useful diagnostic investigation. As an added advantage lung scanning may be carried out at the same time. Unfortunately, the equipment is expensive and not readily available. While any radiation, however small the dose, is undesirable in pregnancy, its risks must be balanced against those of a wrong diagnosis and unnecessary treatment with anticoagulants; and radionuclide venography does seem likely to present little radiation risk to the fetus. When the clinical findings and the results of the available diagnostic tests suggest venous thrombosis, anticoagulant treatment will be necessary for the remainder of pregnancy and for six weeks after delivery. If the investigations give negative results anticoagulant treatment may be discontinued, but the patient should be carefully observed for the rest of pregnancy.

Prophylactic anticoagulants should be considered in women with a combination of serious risk factors, such as thromboembolism in a previous pregnancy or while taking an oral contraceptive, advanced age, high parity, a planned caesarean section, obesity, immobilisation, diabetes mellitus, cardiac disease, hypertension, or bad varicose veins. Such treatment, however, is rarely indicated before 36 weeks.

The next problem is the choice of anticoagulant drug. In treating acute thromboembolism heparin is the drug of choice, but when long-term treatment or prophylaxis is desirable during pregnancy the choice is between oral anticoagulants and heparin. Oral anticoagulants are safe for the mother but they have a low molecular weight and readily cross the placenta to the fetal circulation. Low concentrations of clotting factors predispose to fetal haemorrhage, especially at delivery, and about $15^{\circ}$ of fetuses are estimated to die from this complication. ${ }^{3}$ There is also a small but definite risk from teratogenesis if oral anticoagulants are given during the first trimester. ${ }^{3}$ Heparin does not cross the placental barrier and may be given throughout pregnancy by self-administered subcutaneous injections. ${ }^{36}$ The disadvantages of heparin are its high cost and the inconvenience and discomfort for the patient. Heparin treatment is best controlled by estimation of the plasma heparin concentration, which should be maintained between 0.05 and $0.3 \mathrm{IU} / \mathrm{ml}$; for low-dosage prophylaxis $500-7500 \mathrm{IU}$ of sodium heparin 12-hourly is usually adequate. ${ }^{3}$ When labour starts treatment with heparin can be temporarily suspended. Spinal or epidural anaesthesia is best avoided.

For the sake of the fetus, then, heparin should be the drug of choice for anticoagulant treatment during pregnancy. For those women who cannot tolerate the injections a reasonable compromise is to prescribe heparin in the first trimester, warfarin from 12 until 36 weeks, and heparin thereafter to term. Anticoagulant treatment should be continued for six weeks after delivery, and oral anticoagulants are probably best avoided in the puerperium if the mother is breast-feeding. Patients with prosthetic valves who are taking prolonged oral anticoagulants present a special case, and cardiologists are justifiably reluctant to change anticoagulant drugs. Nevertheless, on current evidence carefully controlled treatment with heparin offers the best chance of a successful outcome to the pregnancy.

${ }^{1}$ Department of Health and Social Security, Report on Confidential Enquiries into Maternal Deaths in England and Wales, 1970-1972. London, HMSO, 1975.

2 Kakkar, V V, and Corrigan, T P, Progress in Cardiovascular Diseases, 1974, 17, 207.

${ }^{3}$ Bonnar, $\mathrm{J}$, in Recent Advances in Obstetrics and Gynaecology, 12, ed J Stallworthy and G Bourne. Edinburgh, Churchill Livingstone, 1977.

4 Browse, N L, British Medical Bulletin, 1978, 34, 163.

${ }^{5}$ Ennis, J T, and Elmes, R J, Radiclogy, 1977, 125, 441.

${ }^{6}$ Spearing, G, et al, British Medical fournal, 1978, 1, 1457. 